\title{
Real-time martensitic transformation kinetics in maraging steel under high magnetic fields
}

\author{
D. San Martin a , N.H. van Dijk ${ }^{\text {b,* }}$, E. Jiménez-Melero ${ }^{\text {b }}$, E. Kampert ${ }^{c}$, U. Zeitler $^{c}$, S. van der Zwaag ${ }^{\text {d }}$ \\ a MATERALIA Group, Department of Physical Metallurgy, Centro Nacional de Investigaciones Metalúrgicas (CENIM-CSIC), Av. Gregorio del Amo 8, 28040 Madrid, Spain \\ ${ }^{\mathrm{b}}$ Fundamental Aspects of Materials and Energy, Faculty of Applied Sciences, Delft University of Technology, Mekelweg 15, 2629 JB Delft, The Netherlands \\ ${ }^{c}$ High Field Magnet Laboratory, Institute for Molecules and Materials, Radboud University Nijmegen, Toernooiveld 7, 6525 ED Nijmegen, The Netherlands \\ ${ }^{\mathrm{d}}$ Novel Aerospace Materials, Faculty of Aerospace Engineering, Delft University of Technology, Kluyverweg 1, 2629 HS Delft, The Netherlands
}

\section{A R T I C L E I N F O}

\section{Article history:}

Received 22 March 2010

Received in revised form 23 April 2010

Accepted 26 April 2010

\section{Keywords:}

Martensitic transformations

Maraging steel

Kinetics

Nucleation

Magnetization measurements

High magnetic field

\begin{abstract}
A B S T R A C T
We have monitored the isothermal transformation kinetics of the austenite phase into the martensite phase in a metastable austenitic maraging steel by time-dependent magnetization measurements for temperatures from 4 to $298 \mathrm{~K}$ and continuous applied magnetic fields up to $30 \mathrm{~T}$. The transformation kinetics is shown to be accelerated by several orders of magnitude when high magnetic fields are applied. Analyzing the transformation rate as a function of magnetic field and temperature provides direct insight into the martensite nucleation process.
\end{abstract}

(C) 2010 Elsevier B.V. All rights reserved.

\section{Introduction}

Maraging steels are a modern class of high-strength steels that show an isothermal martensitic phase transformation. The unusual time-dependent formation of martensite at a constant temperature was first observed by Kurdjumov and Maksimova [1,2] in a Fe-Mn alloy and has subsequently been reported for several other systems, such as $\mathrm{Fe}-\mathrm{Ni}-\mathrm{Cr}$ and $\mathrm{Fe}-\mathrm{Ni}-\mathrm{Mn}$ alloys $[3,4]$. In these systems the martensite fraction strongly depends on both the holding time and the temperature below the martensitic transformation temperature. In contrast, for the more common athermal martensitic transformations the martensite fraction is solely governed by the lowest temperature reached.

Several models have tried to reproduce the experimental $C$ curve kinetics associated to the isothermal formation of martensite [5-7], but have so far not been able to unravel the martensite nucleation mechanism in detail. Experimentally it was found that the martensite formation is not only a function of time and temperature, but can also be induced by e.g. pulsed magnetic fields $[8,9]$. In

\footnotetext{
* Corresponding author at: Fundamental Aspects of Materials and Energy, Department of Radiation, Radionuclides \& Reactors, Faculty of Applied Sciences, Delft University of Technology, Mekelweg 15, 2629 JB Delft, The Netherlands. Tel.: +31 152786775; fax: +31 152788303.

E-mail address: N.H.vanDijk@tudelft.nl (N.H. van Dijk).
}

the present paper we will show how continuous magnetic fields up to $30 \mathrm{~T}$ were used to accelerate the isothermal transformation from austenite to martensite and to in situ monitor the real-time kinetics of the diffusionless martensite formation. Other studies recently focussed on the influence of high magnetic fields on diffusional solid-state phase transformations in steel [10-16].

\section{Time-resolved magnetization measurements}

We have performed time-resolved magnetization measurements for temperatures from 4 to $298 \mathrm{~K}$ in continuous magnetic fields up to $30 \mathrm{~T}$. Fig. 1 summarizes our results at a fixed temperature of $233 \mathrm{~K}$. These experiments were performed with an extraction magnetometer on samples of dimensions $0.5 \mathrm{~mm} \times 3 \mathrm{~mm} \times 3 \mathrm{~mm}$. The composition of the studied metastable austenitic maraging steel amounts to $12 \mathrm{Cr}, 9 \mathrm{Ni}, 4 \mathrm{Mo}, 2 \mathrm{Cu}, 1 \mathrm{Ti}$, $0.7 \mathrm{Al}, 0.3 \mathrm{Mn}, 0.3 \mathrm{Si},<0.01 \mathrm{C}, \mathrm{N}$ and balance Fe (in wt.\%). For this steel the formation of martensite is accompanied by an increase in volume of $\Delta V / V \approx 3 \%$, illustrated by the surface modification shown in the inset of Fig. 1.

During the martensitic transformation the paramagnetic austenite phase $\gamma$ transforms progressively into the ferromagnetic martensite phase $\alpha^{\prime}$. Additionally, a paramagnetic chi-phase $\chi\left(\mathrm{Fe}_{36} \mathrm{Cr}_{12} \mathrm{Mo}_{10}\right)$ [17] with a constant volume fraction of $f_{\chi} \approx 5 \%$ is present in the form of fine precipitates [18]. The transformation 


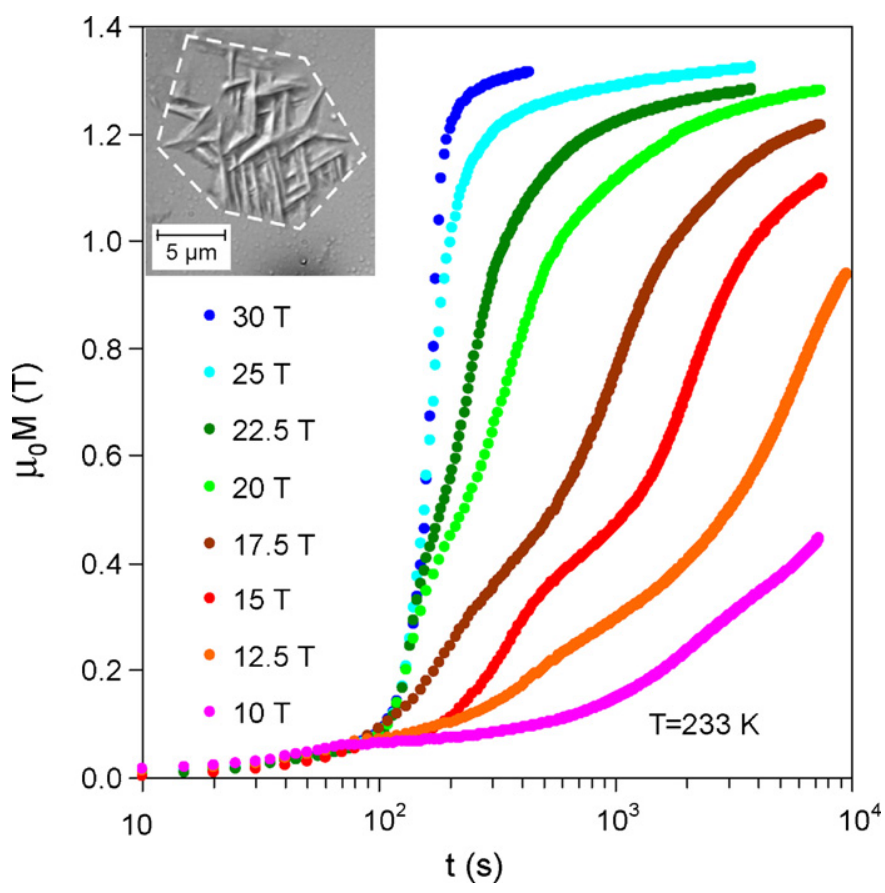

Fig. 1. Magnetization $\mu_{0} M$ versus time $t$ of the metastable austenitic steel in applied magnetic fields from 10 to $30 \mathrm{~T}$ at a fixed temperature of $233 \mathrm{~K}$. The field is increased at a constant ramp rate of $10 \mathrm{~T} / \mathrm{min}$ until the maximum selected field was reached. An isothermal transformation is observed from the paramagnetic austenite phase to the ferromagnetic martensite phase. The inset shows the surface modification caused by stress relief of the transformed martensite plates [18].

results in a strong increase of the sample magnetization $M$. For our specific multiphase material $M$ can be described as a superposition of its constituents' magnetization:

$M=f_{\alpha^{\prime}} M_{\mathrm{s}, \alpha^{\prime}}+f_{\gamma} \chi_{\gamma} H+f_{\chi} \chi_{\chi} H$

where $H$ is the applied magnetic field, $M_{\mathrm{s}, \alpha^{\prime}}$ is the saturation magnetization of the newly formed martensite phase, $\chi_{\gamma}$ is the magnetic susceptibility of the transforming austenite phase, $\chi_{\chi}$ is the magnetic susceptibility of the stable chi-phase, and $f_{\mathrm{i}}$ are the volume fractions of the three phases with $f_{\alpha^{\prime}}+f_{\gamma}+f_{\chi}=1$. A comparison of $d M / d H$ during the linear field ramps at the start and the end of the experiment allows for an independent determination of the paramagnetic susceptibility of the transforming austenite phase and the stable chi-phase. As no significant transformation is observed for fields up to $15 \mathrm{~T}$ during the field ramp, the sample susceptibility amounts to $d M / d H=f_{\gamma} \chi_{\gamma}+f_{\chi} \chi_{\chi}$ in this field range $\left(\chi_{\gamma}, \chi_{\chi}\right.$ and $M_{\mathrm{s}, \alpha^{\prime}}$ are field independent). At $T=233 \mathrm{~K}$ we find $\chi_{\gamma}=0.0035(1)$ and $\chi_{\chi}=0.022(3)$. After removing the paramagnetic contribution, the magnetization data can be transformed directly into the martensite fraction $f_{\alpha^{\prime}}$ by normalization with the saturation magnetization of martensite. We have used the experimental room-temperature value of $\mu_{0} M_{\mathrm{s}, \alpha^{\prime}}=1.48 \mathrm{~T}$ [18] and scaled its temperature dependence according to the relative temperature dependence for pure iron [19].

At the start of the magnetization measurements the magnetic field was increased at a constant sweep rate of $10 \mathrm{~T} / \mathrm{min}$ until the maximum field was reached. Part of the austenite phase was already transformed during this initial field ramp and therefore the experimentally measured transformation time is not representative for the transformation time at the final constant magnetic field. However, since the time to transform a fixed fraction was found to drop exponentially with the applied magnetic field we can remove the effect of this initial transformation by reasonably supposing that the time constant for the transformation scales as $\tau=\tau_{0} \exp \left(-c \mu_{0} H / T\right)$, where $c=89(2) \mathrm{K} / \mathrm{T}$ is an experimental value
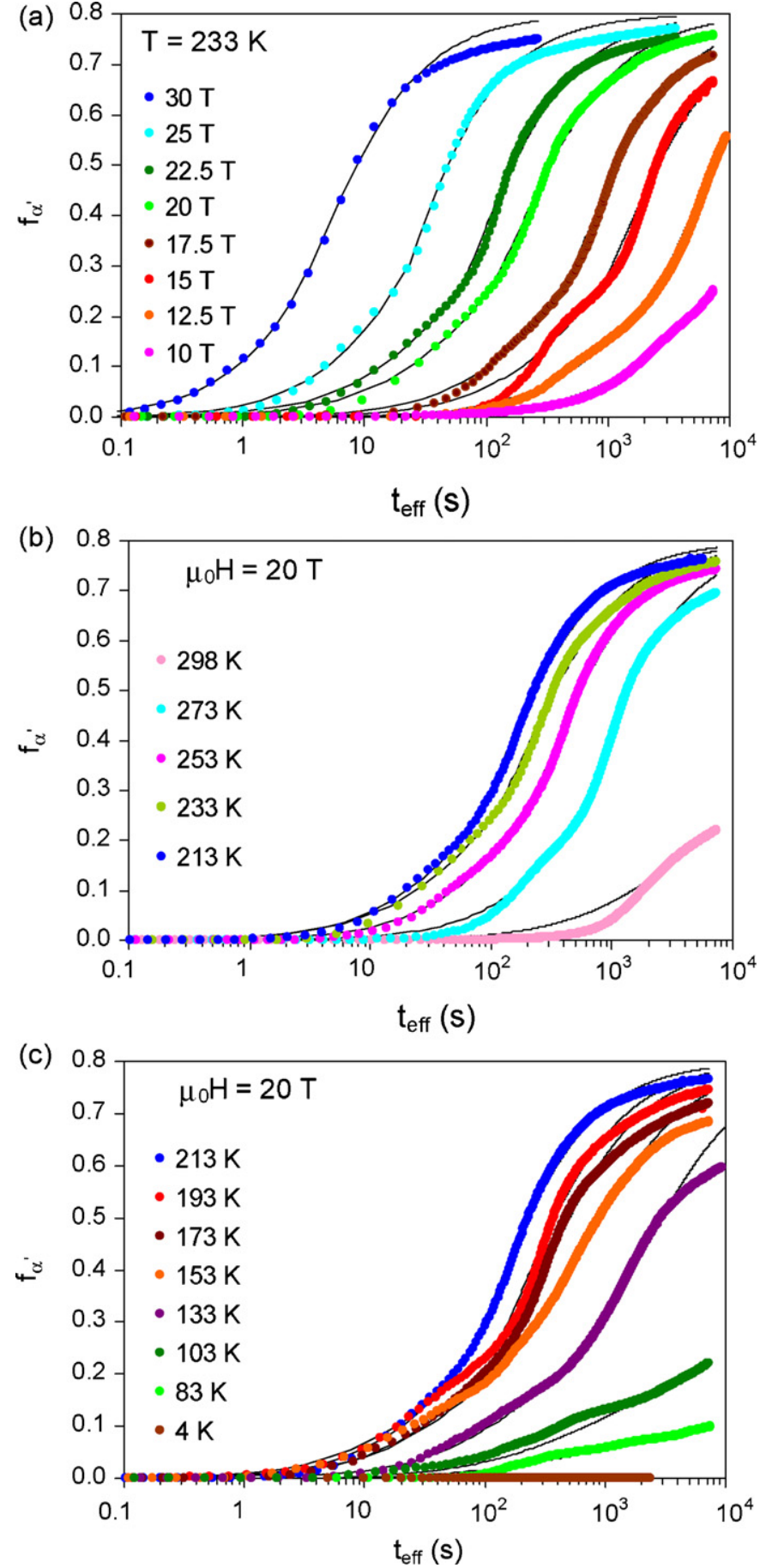

Fig. 2. Volume fraction of martensite $f_{\alpha^{\prime}}$ as a function of the effective time $t_{\text {eff }}$ at the set applied magnetic field for (a) different fields at $T=233 \mathrm{~K}$, (b) different temperatures $T \geq 213 \mathrm{~K}$ and (c) different temperatures $T \leq 213 \mathrm{~K}$ in an applied magnetic field of $\mu_{0} H=20 \mathrm{~T}$. The effective time is obtained by a correction for the field increase at the initial stage of the experiment and the solid lines are a fit to the data (see text).

for our system. In this case the effective transformation time at a constant field $H_{0}$ can be derived by scaling the time elapsed during the initial magnetic field sweep by a factor $[1-\exp (-x)] / x$ with $x=c \mu_{0} H_{0} / T$ (see Appendix A).

\section{Austenite to martensite transformation kinetics}

With this approach the sample magnetization can now be translated into the transformed martensite fraction as a function of the 

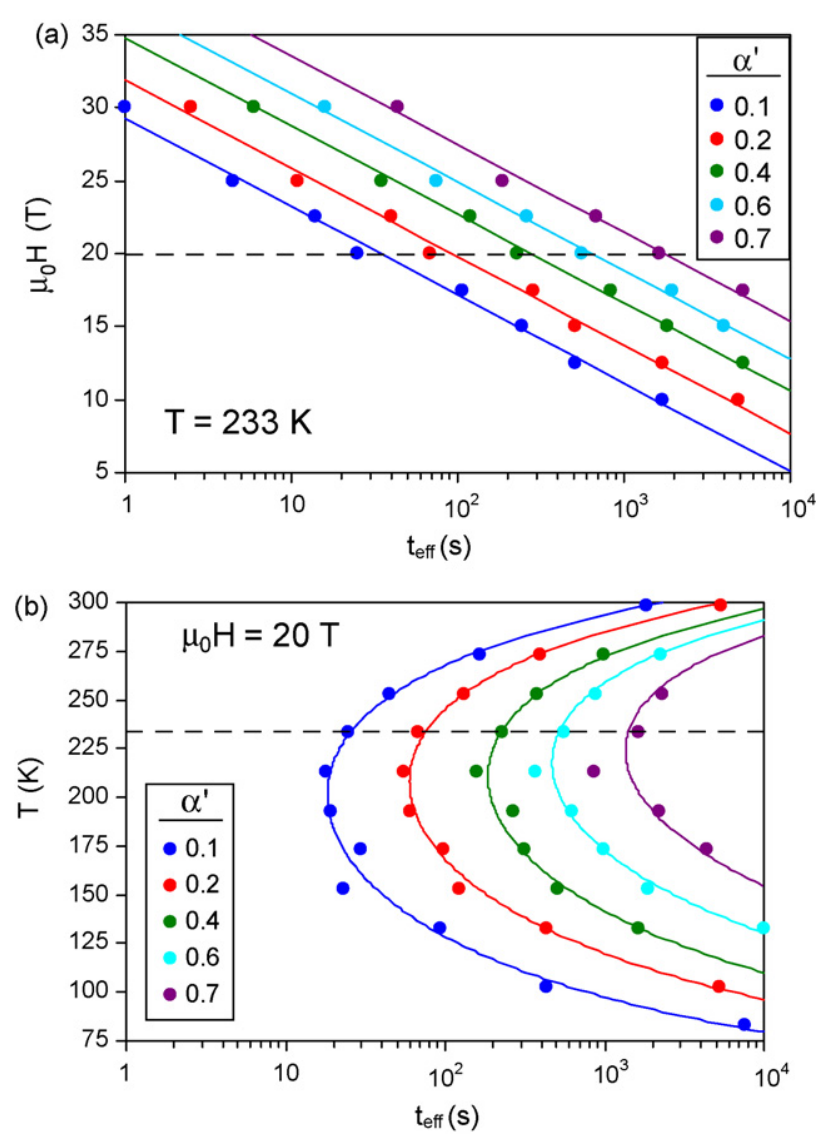

Fig. 3. Time to form a constant martensite volume fraction for (a) the measurements at a constant temperature of $T=233 \mathrm{~K}$ and variable applied magnetic fields and (b) the measurements in a constant applied magnetic field of $\mu_{0} H=20 \mathrm{~T}$ and variable temperatures. The solid lines are a fit to the data (see text).

effective transformation time ( $\left.t_{\text {eff }}\right)$ at a constant applied magnetic field. As shown in Fig. 2, the transformation shows a cross-over behaviour for a fraction of about $25 \%$ and reaches a maximum, field independent, volume fraction of about $80 \%$. Remarkably, the transformation rate increased by orders of magnitude when magnetic fields up to $30 \mathrm{~T}$ were applied, whereas the transformation at $T=233 \mathrm{~K}$ (the temperature where the zero-field transformation rate was reported to be maximized [4]) took about 2 months to complete in zero field [18], only a few minutes were required to reach a final martensite fraction of about $80 \%$ in a magnetic field of $30 \mathrm{~T}$.

\section{Discussion}

In Fig. 3a we have plotted the time to transform a volume fraction of $10,20,40,60$ and $70 \%$ as a function of the magnetic field at a temperature of $T=233 \mathrm{~K}$. The field dependence of the transformation time to form a fixed martensite fraction is described by a single exponential scaling factor (the experimental constant $c$ ) indicating a linear reduction in the energy barrier for nucleation $\Delta G^{*}$ with applied magnetic field $H$.

In Fig. 3b we show the temperature dependence of the transformation time at a fixed magnetic field of $20 \mathrm{~T}$. The observed C-shaped curves can be described by two independent terms: the driving force for nucleation and a mobility term. As martensite growth is extremely fast, the kinetics for the isothermal formation of martensite is purely controlled by the nucleation rate:

$\frac{d f_{\alpha^{\prime}}}{d t}=V_{\alpha^{\prime}} \frac{d N_{\alpha^{\prime}}}{d t}$

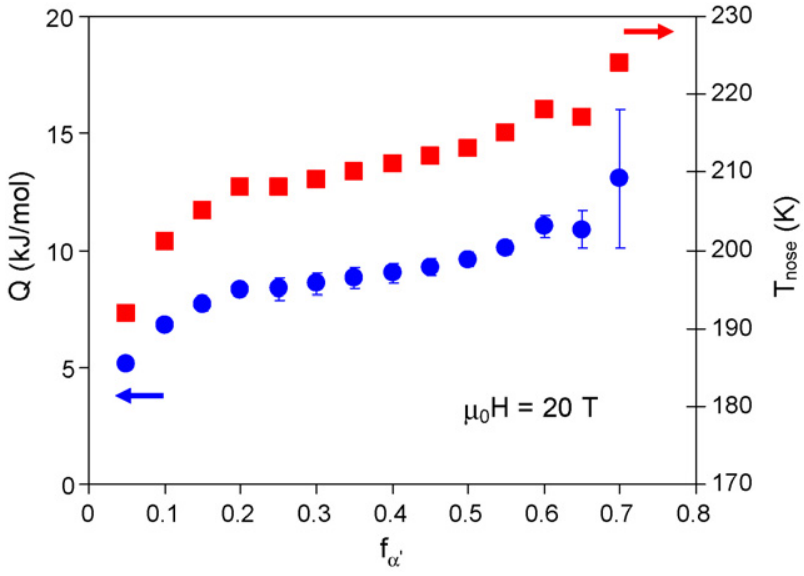

Fig. 4. Temperature at which a maximum transformation rate is observed $T_{\text {nose }}$ (red squares) and the activation energy $Q$ (blue spheres) as a fraction of the martensite volume fraction $f_{\alpha^{\prime}}$ in an applied magnetic field of $20 \mathrm{~T}$ obtained from a fit to the data (see text).

where $V_{\alpha^{\prime}}$ is the average volume of the martensite plates and $d N_{\alpha^{\prime}} / d t$ is the nucleation rate of the martensite plates. The nucleation rate is controlled by two competing exponential contributions:

$\frac{d N_{\alpha^{\prime}}}{d t}=N_{0} v \exp \left(-\frac{\Delta G^{*}(T, H)}{R T}\right) \exp \left(-\frac{Q}{R T}\right)$

The first exponential term is the driving force for the nucleation with $\Delta G^{*}$ referring to the energy barrier for nucleation and the second exponential term is a mobility term with $Q$ the activation energy. The driving force causes a strong acceleration at lower temperatures, while the mobility term causes a deceleration, resulting in the characteristic $C$-curve for the isothermal martensite transformation kinetics. The prefactor $N_{0}$ is the concentration of potential nucleation sites and $v$ is the attempt frequency. The main aim of this study is to probe the transformation kinetics directly over a wide range of temperatures and applied magnetic fields and thereby characterizing the energy barrier for the nucleation of martensite $\Delta G^{*}$. The energy barrier for nucleation can generally be expressed as $\Delta G^{*}=\frac{\Psi}{\Delta g_{v}^{n}} \propto\left|T_{0}(H)-T\right|^{-n}$, where $\Psi$ is a constant and $\Delta g_{v} \propto\left|T_{0}(H)-T\right|$ is the energy gain (per unit volume) of the nucleating phase, which scales with the undercooling with respect to the field-dependent transformation temperature $T_{0}(H)$ [20]. The effective driving force for nucleation $\Delta g_{v}=\Delta g_{v}^{\text {chem }}+\Delta g_{v}^{\text {strain }}+\Delta g_{v}^{\text {mag }}$ is controlled by the difference in chemical potential between both phases $\Delta g_{v}^{\text {chem }}$, the strain energy $\Delta g_{v}^{\text {strain }}$ associated with the volume difference between the austenite and the martensite phase $(\Delta V / V \approx 3 \%$ ) and, in the presence of an applied magnetic field, the change in magnetic energy $\Delta g_{v}^{\mathrm{mag}}$.

A fitting of the temperature dependence of the transformation times at $20 \mathrm{~T}$ reveals that $n=2$ for our system. It is interesting to note that an index of $n=4$ is predicted by the classical nucleation theory for martensite plate nucleation [20-22]. This prediction has never been confirmed experimentally and is clearly contradicted by our present experiments. The fitted martensitic transformation temperature amounts to $T_{0}=411(24) \mathrm{K}$ in a field of $20 \mathrm{~T}\left(d T_{0} / d \mu_{0} H=1.7(1) \mathrm{K} / \mathrm{T}\right)$, with a corresponding value of $\Delta G^{*}\left|1-T / T_{0}(H)\right|^{2}=1.9(1) \mathrm{kJ} / \mathrm{mol}$. At a temperature of $T=233 \mathrm{~K}$ the energy barrier for nucleation $\Delta G^{*}$ is found to be reduced from $15 \mathrm{~kJ} / \mathrm{mol}$ in zero field to $10 \mathrm{~kJ} / \mathrm{mol}$ in a field of $20 \mathrm{~T}$ as a result of the increase in $T_{0}$ from $377 \mathrm{~K}$ in zero field to $411 \mathrm{~K}$ in a field of $20 \mathrm{~T}$. Using the estimated zero field chemical driving force of $V_{m} \Delta g_{v}^{\text {chem }} \approx$ $-3.1 \mathrm{~kJ} / \mathrm{mol}$ at $T=233 \mathrm{~K}$ (with $V_{m} d \Delta g_{\nu}^{\text {chem }} / d T \approx 6.5 \mathrm{~J} / \mathrm{mol} \mathrm{K}$ ) [18] we find $V_{m} \Delta g_{v}^{\text {strain }} \approx 2.2 \mathrm{~kJ} / \mathrm{mol}$ for the strain energy. In an applied 
magnetic field the magnetic energy amounts to $V_{m} \Delta g_{v}^{\operatorname{mag}} / \mu_{0} H=$ $-V_{m} M_{\mathrm{s}, \alpha^{\prime}} \approx-8.44 \mathrm{~J} / \mathrm{molT}$, where $V_{\mathrm{m}}=6.8 \times 10^{-6} \mathrm{~m}^{3} / \mathrm{mol}$ is the molar volume and $M_{\mathrm{s}, \alpha^{\prime}}$ the saturation magnetization of the martensite phase.

As shown in Fig. 4 the activation energy $Q$ is found to range from 5 to $10 \mathrm{~kJ} / \mathrm{mol}$, depending on the fraction transformed. This variation in $Q$ is also reflected in the temperature $T_{\text {nose }}$ for which the maximum transformation rate is observed. The obtained values for $Q$ are small compared to the activation energy for the movement of individual atoms in austenite $(284 \mathrm{~kJ} / \mathrm{mol})$ [23], but in good agreement with previous studies on similar austenitic steels [24], which proposed that the movement of dislocations is the rate limiting step for martensite nucleation at low temperatures. The experimental observation that $Q$ depends on the fraction transformed in this system indicates that the mobility of dislocations, required for the nucleation of martensite plates, is affected significantly by the transformation strain accumulating in the untransformed austenite matrix.

The overall transformation kinetics of this system is well described by:

$\frac{d f_{\alpha^{\prime}}}{d t}=\frac{\left(f_{0}-f_{\alpha^{\prime}}\right)^{2}}{\tau} \propto\left(f_{0}-f_{\alpha^{\prime}}\right)^{2} \exp \left(-\frac{Q\left(f_{\alpha^{\prime}}\right)}{R T}\right)$

where $f_{0} \approx 80 \%$ is the maximum transformable fraction and $\tau=$ $\left(1 / V_{\alpha^{\prime}} N_{0} v\right) \exp \left(\left(\Delta G^{*}+Q\right) / R T\right)$ is the time constant of the system (at $f_{\alpha^{\prime}}=0$ ). This model results from a linear scaling of the number of potential nucleation sites $N_{0} \propto f_{0}-f_{\alpha^{\prime}}$ and the average volume of the martensite plates $V_{\alpha^{\prime}} \propto f_{0}-f_{\alpha^{\prime}}$ with the untransformed phase fraction. The maximum achievable fraction of $80 \%$ is probably caused by strain accumulation in the untransformed austenite that effectively reduces $\Delta g_{v}$ for a progressive transformation. As shown in Fig. 2, the data are well represented by the model when $Q$ is assumed to increase linearly with the fraction transformed until a fraction of $25 \%$ is reached, while for higher fractions a constant value is found. Finally, it is interesting to note that no significant texture was observed for the martensite plates when the samples were transformed in high magnetic fields.

\section{Conclusions}

In summary, we have monitored the isothermal transformation kinetics of the austenite phase into the martensite phase in a metastable austenitic maraging steel by time-dependent magnetization measurements for temperatures from 4 to $298 \mathrm{~K}$ and continuous applied magnetic fields up to $30 \mathrm{~T}$. The main conclusions are:

1. Exploiting that the ferromagnetic martensite phase is formed from the paramagnetic austenite phase enabled us to monitor the time dependence of the martensite fraction. A careful analysis of the data allowed us to access a wide range of transformation times from 0.1 to $10^{4} \mathrm{~s}$.

2. The transformation kinetics is shown to be accelerated by several orders of magnitude when high magnetic fields are applied.

3. The temperature dependence of the transformation kinetics allowed us to establish the temperature dependence of the energy barrier for martensite nucleation.

4. The activation energy for the mobility of dislocations was found to gradually increase as the transformation proceeds. This indicates a clear link between the dislocation mobility and the evolving microstructure.

\section{Acknowledgments}

This work was financially supported in part by the Foundation for Fundamental Research on Matter (FOM) of the Netherlands Organisation for Scientific Research (NWO) and the Netherlands Institute for Metals Research (NIMR) currently known as the Materials Innovation Institute (M2i).

\section{Appendix A. Appendix}

As indicated in Eq. (4) the transformation kinetics is controlled by a time constant $\tau$. This time constant depends on both the temperature $T$ and the applied magnetic field $\mu_{0} H$ :

$\tau=\tau_{0} \exp \left(-c \frac{\mu_{0} H}{T}\right)$

In the isothermal transformation measurements we have applied a linear increase in field until the maximum field $\mu_{0} H_{0}$ is reached. For high magnetic fields $\left(\mu_{0} H>15 \mathrm{~T}\right)$ a significant fraction is transformed during the time the field is still increasing.

As the fraction transformed is controlled by the scaled time $\mathrm{t} / \tau$, the isothermal transformation kinetics at constant field can be obtained when we replace the measured transformation time at varying field $t$ by an effective time at constant field $t_{\text {eff. }}$. A time integration of $1 / \tau$ performed for the time to reach the maximum field $\mu_{0} H_{0}$ during the linear field $\operatorname{ramp}(\Delta t)$ is therefore equivalent to a time integration of $1 / \tau$ performed for an effective transformation time at constant field $\left(\Delta t_{\text {eff }}\right)$ :

$\int_{0}^{\Delta t} \frac{d t}{\tau\left(H=\frac{t}{\Delta t} H_{0}\right)}=\int_{0}^{\Delta t_{e f f}} \frac{d t}{\tau\left(H=H_{0}\right)}$

The integral for the actual time (linearly increasing field)

$$
\begin{aligned}
\int_{0}^{\Delta t} \frac{d t}{\tau\left(H=\frac{t}{\Delta t} H_{0}\right)} & =\frac{1}{\tau_{0}} \int_{0}^{\Delta t} \exp \left(c \frac{\mu_{0} H_{0}}{T} \frac{t}{\Delta t}\right) d t \\
& =\frac{\Delta t}{\tau_{0}} \frac{\exp \left(c \mu_{0} H_{0} / T\right)-1}{\left(c \mu_{0} H_{0} / T\right)}
\end{aligned}
$$

corresponds to the integral for the effective time (constant field)

$$
\begin{aligned}
\int_{0}^{\Delta t_{\text {eff }} \frac{d t}{\tau\left(H=H_{0}\right)}} & =\frac{1}{\tau_{0}} \int_{0}^{\Delta t_{\text {eff }}} \exp \left(c \frac{\mu_{0} H_{0}}{T}\right) d t \\
& =\frac{\Delta t_{\text {eff }}}{\tau_{0}} \exp \left(c \frac{\mu_{0} H_{0}}{T}\right)
\end{aligned}
$$

A comparison leads to an expression for the effective transformation time:

$\frac{\Delta t_{\text {eff }}}{\Delta t}=\frac{\left[1-\exp \left(-c \mu_{0} H_{0} / T\right)\right]}{c \mu_{0} H_{0} / T}$

\section{References}

[1] G.V. Kurdjumov, O.P. Maksimova, Dokl. Akad. Nauk SSSR 61 (1948) 83.

[2] G.V. Kurdjumov, O.P. Maksimova, Dokl. Akad. Nauk SSSR 73 (1950) 95.

[3] T. Kakeshita, K. Kuroiwa, K. Shimizu, T. Ikeda, A. Yamagishi, M. Date, Mater. Trans. JIM 34 (1993) 415.

[4] M. Holmquist, J.-O. Nilsson, A. Hultin Stigenberg, Scripta Metall. Mater. 33 (1995) 1367.

[5] T. Kakeshita, K. Kuroiwa, K. Shimizu, T. Ikeda, A. Yamagishi, M. Date, Mater. Trans. JIM 34 (1993) 423.

[6] G. Ghosh, G.B. Olson, Acta Metall. Mater. 42 (1994) 3371.

[7] A. Borgenstam, M. Hillert, Acta Mater. 48 (2000) 2777.

[8] T. Kakeshita, K. Shimizu, S. Funada, M. Date, Acta Metall. 33 (1985) 1381.

[9] T. Fukuda, T. Kakeshita, K. Kindo, Mater. Sci. Eng. A 438-440 (2006) 212.

[10] H.D. Joo, S.U. Kim, N.S. Shin, Y.M. Koo, Mater. Lett. 43 (2000) 225. 
[11] H. Guo, M. Enomoto, Mater. Trans. JIM 41 (2000) 911.

[12] M. Enomoto, H. Guo, Y. Tazuke, Y.R. Abe, M. Shimotomai, Metall. Mater. Trans. A 32 A (2001) 445

[13] Y. Zhang, C. He, X. Zhao, C. Esling, L. Zuo, Adv. Eng. Mater. 6 (2004) 310

[14] Y. Zhang, C. He, X. Zhao, L. Zuo, C. Esling, J. Magn. Magn. Mater. 294 (2005) 267.

[15] S. Rivoirard, F. Gaucherand, O. Bouaziz, E. Pinto Da Costa, E. Beaugnon, ISIJ Int. 46 (2006) 1274.

[16] R.A. Jaramillo, S.S. Babu, G.M. Ludtka, R.A. Kisner, J.B. Wilgen, G. MackiewiczLudtka, D.M. Nicholson, S.M. Kelly, M. Murugananth, H.K.D.H. Bhadeshia, Scripta Mater. 52 (2005) 461.

[17] J.K.L. Lai, C.H. Shek, Y.Z. Shao, A.B. Pakhomov, Mater. Sci. Eng. A 379 (2004) 308.
[18] D. San Martin, K.W.P. Aarts, P.E.J. Rivera-Diaz-del-Castillo, N.H. van Dijk, E. Brück, S. van der Zwaag, J. Magn. Magn. Mater. 320 (2008) 1722.

[19] A.S. Arrott, B. Heinrich, J. Appl. Phys. 52 (1981) 2113.

[20] J.W. Christian, The Theory of Transformations in Metals and Alloys, 3rd ed., Pergamon, Oxford, 2002, pp. 1062-1075.

[21] D.A. Porter, K.E. Easterling, M.Y. Sherif, Phase Transformations in Metals and Alloys, 3rd ed., CRC Press, Boca Raton, 2009, pp. 380-384

[22] Q.P. Meng, Y.H. Rong, T.Y. Hsu, Metall. Mater. Trans. A 37A (2006) 1405.

[23] J. Kučera, K. Stránský, Mater. Sci. Eng. 52 (1982) 1.

[24] A. Borgenstam, M. Hillert, Acta Mater. 45 (1997) 651. 